\title{
Effect of cysteamine supplementation during in vitro culture of early stage bovine embryos on blastocyst rate and quality
}

\author{
Martina Lojkic ${ }^{1}$, Iva Getz ${ }^{1}$, Marko Samardzija ${ }^{1}$, Mario Matkovic ${ }^{2}$, Goran Bacic ${ }^{1}$, \\ Tugomir Karadjole ${ }^{1}$, Nino Macesic ${ }^{1}$, Ivan Folnozic ${ }^{1}$, Branimira Spoljaric ${ }^{1}$ \\ ${ }^{1}$ University of Zagreb, Faculty of Veterinary Medicine, Clinic for Obstetrics and Reproduction, Zagreb, Croatia \\ ${ }^{2}$ Center for Reproduction and Animals Breeding of Croatia, Novi Zagreb, Croatia \\ Received January 11, 2012 \\ Accepted May 16, 2012
}

\begin{abstract}
The aim of this study was to evaluate whether the addition of cysteamine to the in vitro culture media enhances the yield, hatching rate, total cell number and inner cell mass/total cell number ratio of bovine embryos. A total of 933 bovine oocytes collected from ovaries of 60 slaughtered donors were subjected to in vitro maturation and in vitro fertilization. Following fertilization, embryos were cultured in synthetic oviductal fluid without glucose. After $24 \mathrm{~h}$ embryos were transferred into synthetic oviductal fluid with $1.5 \mathrm{mM}$ glucose and 0 (control), 50, 100 and $200 \mu \mathrm{M}$ of cysteamine. After $48 \mathrm{~h}$, the embryos were transferred into synthetic oviductal fluid with glucose but without cysteamine and cultured until Day 9. The number of cleaved embryos on Day 2, the total number of blastocysts on Day 7 and the number of hatched blastocysts on Day 9 were calculated. Differential staining of inner cell mass and trophectoderm cells of blastocysts were performed on Day 7 and Day 9 of in vitro culture. Supplementation of in vitro culture media with $100 \mu \mathrm{M}$ cysteamine increased the blastocyst yield $(P<0.05)$ without affecting the hatching rate. Furthermore, the embryos cultured in the presence of $100 \mu \mathrm{M}$ cysteamine had significantly higher number of inner cell mass cells $(P<0.05)$ and the proportion of inner cell mass cells $(P<0.05)$ compared with the controls. The results of the present study demonstrated that the addition of $100 \mu \mathrm{M}$ cysteamine to the in vitro culture media improved blastocyst production rate and enhance embryo quality, which could lead to the improvement of the in vitro culture system for bovine embryos.
\end{abstract}

Oxidative stress, antioxidants, embryo yield, differential staining, total cell number

Oocytes and embryos are protected against oxidative stress by oxygen scavengers that are normally present in follicular and oviductal fluids. In an in vitro production system, oocytes and embryos are removed from their natural environment and this defense system is lost (Wang et al. 2002). Oxidative stress induces mitochondrial dysfunction, DNA, RNA and protein damage (Comporti 1989), inhibition of sperm-oocyte fusion (Aitken et al. 1993), embryo cell block and apoptosis, especially at the blastocyst stage (Parchment 1991). In order to improve in vitro embryo production, oxidative stress must be controlled during in vitro culture. The addition of various antioxidants to the in vitro culture media may protect oocytes and embryos from the detrimental effect of oxygen-derived free radicals (Del Corso et al. 1994).

Glutathione $(\mathrm{GSH})$ is a major non-protein sulphhydril compound in mammalian cells which protects the cell from oxidative damage. Synthesis of glutathione depends on the availability of cysteine in the media (Furnus and De Matos 1999). Cysteamine is a low molecular-weight thiol compound that may reduce cystine to cysteine and enhance oocyte glutathione synthesis (Issels et al. 1988). It was demonstrated that the addition of cysteamine to the in vitro maturation media improves the rate of embryo development by increasing glutathione synthesis in cow and sheep (de Matos et al. $1996 ; 2002 a)$. The benefits of supplementing the in vitro maturation medium (IVM) with

Address for correspondence:

Dr. Martina Lojkic, PhD

Clinic for Obstetrics and Reproduction

Faculty of Veterinary Medicine University of Zagreb

Heinzelova 55, 10000 Zagreb, Croatia
Phone: +385 (1) 2390326

Fax: +385 (1)2441 390

E-mail: martinakaradjole@gmail.com

http://actavet.vfu.cz/ 
cysteamine are reviewed by Deleuze and Goudet (2010). However, it is still unclear if supplementation with antioxidants is required during in vitro culture of embryos due to low oxygen concentration. It was reported that the addition of antioxidants to the in vitro culture improved blastocyst production (Takahashi et al. 1993; Olson and Seidel 2000; de Matos et al. 2002b). Furthermore, as the lowest glutathione content was found in 2- to 8-cell embryos, the early cleavage stage embryos are, therefore, probably more sensitive to oxidative stress than the other stages.

The aim of this study was to evaluate the effect of cysteamine supplementation to the in vitro culture media during early stages of development on the embryo developmental rate and quality by means of differential staining.

\section{Materials and Methods}

Reagents and media

Unless otherwise stated, all chemicals used in this study were purchased from Sigma Chemical Co. (SigmaAldrich Chemie GMBH, Germany). The oocyte maturation medium was TCM 199 bicarbonate medium supplemented with 10\% foetal calf serum (FCS) (GIBCO Invitrogen Corporation, Auckland, New Zealand), follicle stimulating hormone and luteinizing hormone (Pergonal, 75/75 IU, Serono), $1 \mu \mathrm{g} / \mathrm{ml}$ estradiol-17ß and $100 \mu \mathrm{M}$ cysteamine. The in vitro fertilization medium (IVF) was modified by Tyrode's bicarbonate buffered solution supplemented with $10 \mu \mathrm{g} / \mathrm{ml}$ heparin, $5 \mu \mathrm{g} / \mathrm{ml}$ hypotaurine, $5 \mu \mathrm{g} / \mathrm{ml}$ epinephrine and $6 \mathrm{mg} / \mathrm{ml}$ bovine serum albumine (BSA). The embryo culture medium was synthetic oviductal fluid (SOF) with amino acids, $1 \mu \mathrm{M}$ glutamine, $8 \mathrm{mg} / \mathrm{ml} \mathrm{BSA}$ and 0 (SOF0) or $1.5 \mathrm{mM}$ glucose (SOFg). All media were allowed to equilibrate at 39 ${ }^{\circ} \mathrm{C}$ in the atmosphere of $5 \% \mathrm{CO}_{2}$.

In vitro embryo production

A total of 60 bovine ovaries were collected at the abattoir and transported to the laboratory in saline with antibiotics (100 IU penicillin and $100 \mu \mathrm{g}$ streptomycin $/ \mathrm{ml}$ ) at $37^{\circ} \mathrm{C}$ within $3 \mathrm{~h}$ of slaughter. Cumulusoocyte complexes (COCs) were aspirated from 2 to $8 \mathrm{~mm}$ diameter follicles using $18 \mathrm{G}$ needle attached to a vacuum pump. Oocytes with intact cumulus investment and evenly granulated cytoplasm were matured in vitro in groups of 10 in $50 \mu \mathrm{l}$ droplets of maturation medium under mineral oil at $39{ }^{\circ} \mathrm{C}$ with $5 \% \mathrm{CO}$ in air for $24 \mathrm{~h}$. The expanded COCs were washed in HEPES-TALP medium supplemented with $6 \mathrm{mg} / \mathrm{ml} \mathrm{BSA}$ and transferred into $40 \mu \mathrm{l}$ droplets of IVF medium under mineral oil. In all experiments, frozen semen from the same bull was used. Sperm preparation for IVF on BoviPure ${ }^{\circledR}$ (Nidacon Laboratories AB, Gothenberg, Sweden) gradient was accomplished according to Samardzija et al. (2006). Final concentration was adjusted to $1 \times 10^{6} \mathrm{spermatozoa} / \mathrm{ml}$. Incubations were carried out at $39{ }^{\circ} \mathrm{C}$ in $5 \% \mathrm{CO}_{2}$ in air for $24 \mathrm{~h}$. After the sperm-oocytes co-incubation (day $0=$ day of fertilization), the presumptive zygotes were denuded from cumulus cells and spermatozoa and then washed in HEPES-TALP media and SOF0 media. Fertilized oocytes were cultured in vitro in SOF 0 media for $24 \mathrm{~h}$ and then transferred into SOFg with 0, 50, 100 and $200 \mu \mathrm{M}$ cysteamine. Cysteamine was added at Day 2 of in vitro culture (IVC) and the medium was replaced $48 \mathrm{~h}$ later with SOFg without cysteamine. The embryos were cultured in vitro until Day 9 at $39{ }^{\circ} \mathrm{C}$ in $5 \% \mathrm{CO}_{2} 7 \% \mathrm{O}_{2}$ in $88 \% \mathrm{~N}_{2}$. The endpoints for evaluation were Day 2 cleavage rates; Day 7 blastocyst rates; Day 9 hatching rates, total cell number (TCN) and differentiation of the embryonic cells into inner cell mass (ICM) and trophectoderm (TE) cells in Day 7 and Day 9 embryos.

\section{Differential staining of blastocysts}

Hatched blastocysts were used as such and the zona of intact blastocysts was removed by treatment with $0.5 \%$ pronase. Zona-free embryos were washed $\times 5$ in phosphate buffered saline (PBS) containing $0.1 \%$ polyvinyl alcohol (PVA). Embryos were then incubated in a $30: 70$ dilution of rabbit anti-bovine whole serum in TCM 199 bicarbonate at $39^{\circ} \mathrm{C}$ for $1 \mathrm{~h}$. After washing in PBS $0.1 \%$ PVA, the embryos were incubated in a 1: 4 dilution of a guinea pig complement in TCM 199 bicarbonate supplemented with $10 \mu \mathrm{g} / \mathrm{ml}$ propidium iodide (PI) for $1 \mathrm{~h}$. Embryos were then briefly washed in ice-cold TCM 199 Hepes supplemented with $10 \mu \mathrm{g} / \mathrm{ml}$ PI and fixed in ice-cold absolute ethanol. After fixation, the embryos were transferred for 3-5 $\mathrm{min}$ to $10 \mu \mathrm{g} / \mathrm{ml}$ bisbenzimide (Hoechst 33343) in absolute alcohol at room temperature. Presumptive stained blastocysts were transferred to a drop of glycerol on a microscopic slide and covered with a cover slip. Embryos were examined under a fluorescence microscope (Olympus, Tokyo, Japan) equipped with a UV filter. Bisbenzimide-stained ICM nuclei appeared blue and TE nuclei labelled with both bisbenzimide and PI appeared red or pink. The ICM and TE nuclei were counted under the microscope.

\section{Statistical analysis}

The statistical analyses among treatments were done by ANOVA (StatSoft, Statistica, 7.1 version 2005) using the arcsine transformation $(\arcsin \sqrt{ } / 100)$ of the percent values and comparisons by Tukey's post hoc test analysis. $P<0.05$ was considered significant. 


\section{Results}

Effect of cysteamine supplementation during IVC on embryo development

A total of 933 oocytes in 7 independent replicates were submitted to IVM, IVF and IVC to evaluate the effect of cysteamine supplementation to the in vitro culture medium on subsequent embryo development. The blastocyst yields at Day 7 of in vitro culture were significantly higher than the corresponding value for controls following supplementation of in vitro culture medium with $100 \mu \mathrm{M}$ cysteamine $(P<0.05)$, whereas the concentration of 50 and $200 \mu \mathrm{M}$ had no effect (Table 1).

Table 1. Effect of supplementation of in vitro culture media with cysteamine on blastocyst development (mean \pm S.E.M.)

\begin{tabular}{lcccc}
\hline Cysteamine $(\mu \mathrm{M})$ & Oocytes $(\mathrm{n})$ & Day 2 cleavage rate $(\%)$ & Day 7 blastocyst rate $(\%)$ & Day 9 hatching rate $(\%)$ \\
\hline 0 & 234 & $82.49 \pm 3.3$ & $21.94 \pm 3.2$ & $8.89 \pm 2.7$ \\
50 & 229 & $83.21 \pm 3.4$ & $29.17 \pm 2.7$ & $15.12 \pm 3.2^{*}$ \\
100 & 221 & $82.15 \pm 3.7$ & $33.26 \pm 2.2^{*}$ & $13.98 \pm 1.3$ \\
200 & 249 & $82.44 \pm 3.8$ & $21.38 \pm 4.3$ & $7.31 \pm 2.0$ \\
\hline
\end{tabular}

*values within the same column differ significantly from the controls $(P<0.05)$, controls $=0 \mu \mathrm{M}$ cysteamine

Effect of cysteamine supplementation during IVC on embryo quality A random sample of grade 1 blastocysts on day $7(n=42)($ Fig. 2$)$ and all grade 1 hatched blastocyst $(\mathrm{n}=59)$ on Day 9 of in vitro culture (Fig. 3) were selected for differential staining. The total number of cells was significantly affected when $200 \mu \mathrm{M}$ cysteamine was added to in vitro culture both in Day 7 or Day 9 embryos. This concentration reduced the total cell number $(P<0.05)$ compared to controls. However, ICM cell number was

$\square 0 \mu \mathrm{M} \square 50 \mu \mathrm{M} \square 100 \mu \mathrm{M} \square 200 \mu \mathrm{M}$

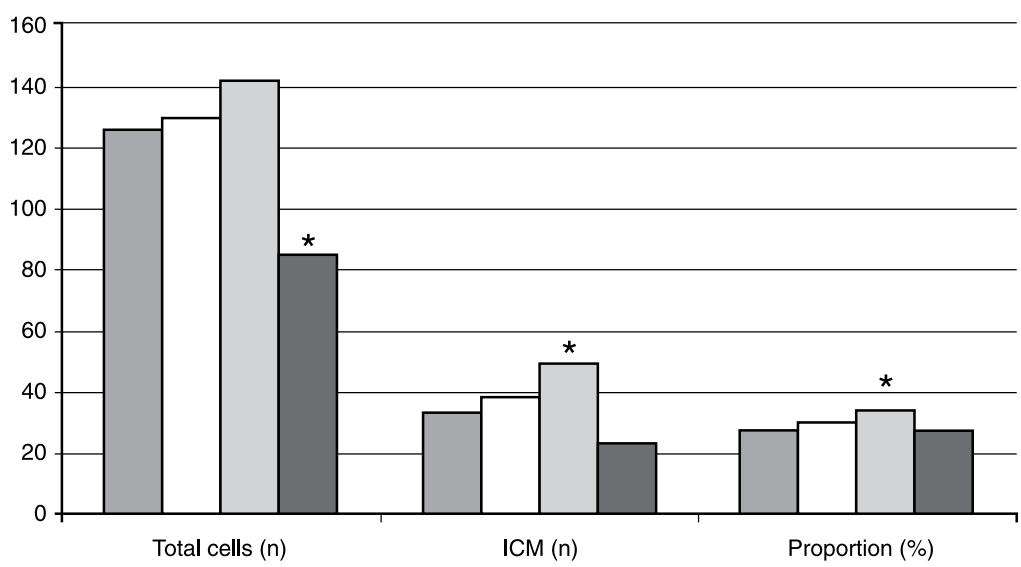

*values differ significantly from the controls $(P<0.05)$, controls $=0 \mu \mathrm{M}$ cysteamine

Fig.1. Mean total cell number, number of inner cell mass cells (ICM) and proportion of inner cell mass in total cell number on Day 7 blastocysts following supplementation of in vitro culture media with 50, 100 and $200 \mu \mathrm{M}$ cysteamine 
$\square 0 \mu \mathrm{M} \square 50 \mu \mathrm{M} \quad \square 100 \mu \mathrm{M} \square 200 \mu \mathrm{M}$

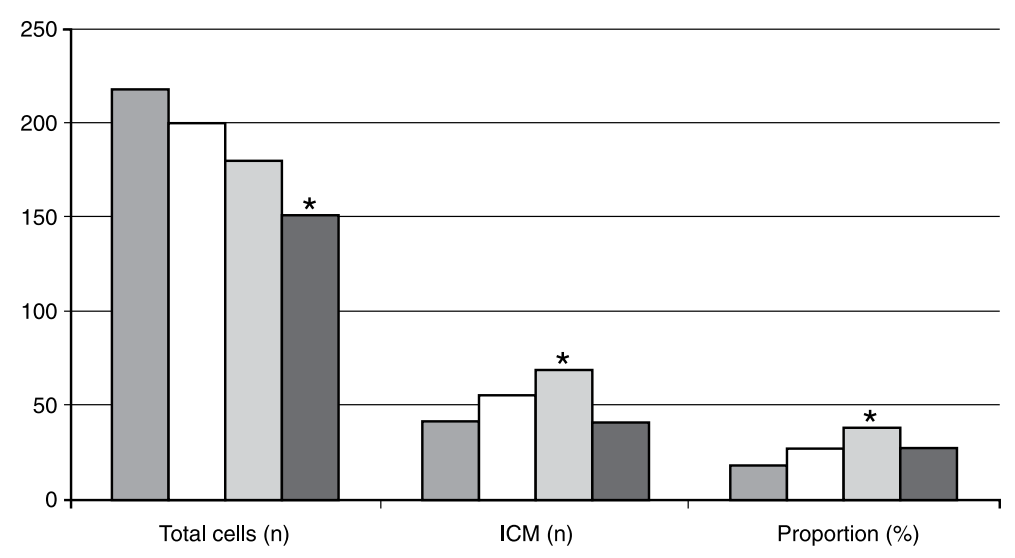

*values differ significantly from the controls $(P<0.05)$, controls $=0 \mu \mathrm{M}$ cysteamine

Fig. 2. Mean total cell number, number of inner cell mass cells (ICM) and proportion of inner cell mass in total cell number in Day 9 hatched blastocysts following supplementation of in vitro culture media with 50, 100 and $200 \mu \mathrm{M}$ cysteamine

the highest in $100 \mu \mathrm{M}$ cysteamine group in both Day 7 blastocysts and Day 9 hatched blastocyst $(P<0.05)$. The same group also displayed significantly higher proportion of ICM cells $(P<0.05)$.

\section{Discussion}

Oxidative stress mediated by reactive oxygen species results in an imbalance of the intracellular redox potential (Balaban et al. 2005). Intracellular glutathione plays a critical role in maintaining the redox state of the oocytes and embryos by protecting cells against oxidative damage. Glutathione is synthesized by $\gamma$-glutamyl cycle, and its biosynthesis depends on the availability of cysteine, a highly unstable aminoacid that is readily oxidized to cystine (Furnus and de Matos 1999). Intracellular glutathione concentration after IVM is a good biological indicator of the cytoplasmic maturation and viability of the oocyte. After the addition of exogenous cysteine during oocyte maturation in vitro, GSH content increases, providing a pool of GSH which are necessary for sperm decondensation, oocyte activation and transformation of fertilizing sperm head into the male pronucleus (Yoshida et al. 1993; Furnus and de Matos 1999). The addition of cysteamine in the maturation media of bovine and sheep oocytes resulted in an increase in GSH levels by reducing oxidized cystine to cysteine in media and an improvement in embryo developmental rate up to the blastocyst stage (de Matos et al. 1996; 2002a). This fact suggests that the beneficial effects of cysteamine on in vitro maturation and subsequent embryo development were mediated by GSH (Isles et al. 1988). This was in agreement with our previous study (Karadjole et al. 2006) which demonstrated that the addition of cysteamine to the in vitro maturation media significantly improved the rate of blastocyst development and the quality of blastocyst by increasing inner cell mass cell number. The intracytoplasmic glutathione concentration varies during preimplatation development of in vitro produced bovine embryos. The lowest level of GSH was found in 2-8 cell embryos as the de novo synthesis of GSH begins to increase at the 9-16-cell stage embryos (Van Langendonckt et al. 1998) following the highest level found in hatched blastocyst (Lim 
et al. 1996). The fact that early stage embryos are probably more sensitive to oxidative stress because of exhausted GSH pool synthesized during IVM inspired us to supplement the in vitro culture media with cysteamine during the first stages of embryo development. The results demonstrated that the addition of $100 \mu \mathrm{M}$ cysteamine during early in vitro development of the embryos significantly improved the development of the blastocyst on Day 7 of in vitro culture compared to controls $(P<0.05)$. This was in agreement with de Matos et al. (2002b) who demonstrated that the addition of cysteamine to maturation media and 2-8-cell bovine embryos results in an increase of embryo quality and blastocyst production. Similarly, the supplementation of cysteamine to both IVM and IVC media improved the blastocyst production rate in buffalo, without compromising their health (Anand et al. 2008). The beneficial effect of cysteamine present during the early stages of embryo development is probably acquired through increased glutathione concentration, which could help overcome the developmental block at 8- to 16-cell stage embryos (Lee et al. 2000) when the GSH stores produced during in vitro maturation are nearly exhausted. Although reactive oxygen species are deleterious to the oocyte and embryo quality, an excess of antioxidant supplementation adversely affects blastocyst development (Guerin at al. 2001; Anand et al. 2008). In our study, supplementation of in vitro culture media with $100 \mu \mathrm{M}$ cysteamine gave the best results, followed by $50 \mu \mathrm{M}$ cysteamine, while $200 \mu \mathrm{M}$ cysteamine was found ineffective regarding the blastocyst yield, but significantly reduced the total cell number in developing embryos $(P<0.05)$. The addition of $100 \mu \mathrm{M}$ cysteamine to the in vitro culture media resulted in a significantly higher ICM cell number and ICM/TCN proportion in Day 7 blastocyst $(P<0.05)$, without affecting the total cell number compared to the control group. Blastocoel fluid contains hydrogen peroxide which is cytotoxic and can induce apoptosis in inner cell mass cells. Treatment with hydrogen peroxide applied to oocytes or embryos decreased the blastocyst development and increased the induction of permanent embryo arrest or apoptosis in a stage-dependent manner (Bain et al. 2011). The increase of inner cell mass cells could, therefore, be explained with the fact that cysteamine addition to the IVC media increases intracellular glutathione content and since glutathione is involved in the removal of hydrogen peroxide, inner cell mass cells would not become apoptotic.

In conclusion, the inclusion of low-molecular-weight thiol compound such as cysteamine to in vitro culture system controls the oxidative stress, improves the blastocyst production rates and enhances embryo quality, which could lead to the improvement of the in vitro culture system.

\section{References}

Aitken RJ, Harkiss D, Buckingham D 1993: Relationship between iron-catalysed lipid peroxidation potential and human sperm function. J Reprod Fertil 98: 257-265

Anand T, Kumar D, Chauhan MS, Manik RS, Palta P 2008: Cysteamine supplementation of in vitro maturation media, in vitro culture media or both media promotes in vitro development of buffalo (Bubalus bubalis) embryos. Reprod Fertil Dev 20: 253-257

Bain NT, Madan P, Betts DH 2011: The early embryo response to intracellular reactive oxygen species is developmentally regulated. Reprod Fertil Dev 23: 561-575

Balaban RS, Nemoto S, Finkel T 2005: Mitochondria, oxidants and aging. Cell 120: 483-495

Comporti M 1989: Three models of free radical induced cell injury. Chem Biol Interact 72: 1-56

de Matos DG, Furnus CC, Moses DF, Martinez AG, Matkovic M 1996: Stimulation of GSH synthesis of in vitro matured bovine oocytes and its effect on embryo development and freezability. Mol Reprod Dev 54: 451-457

de Matos DG, Gasparrini B, Pasqalini SR, Thompson JG 2002a: Effect of glutathione synthesis stimulation during in vitro maturation of ovine oocytes on embryo development and intracellular peroxide content. Theriogenology 57: 1443-1451

de Matos DG, Herrera C, Cortvrindt R, Smitz J, Van Soom A, Nogueira D, Pasqalini SR 2002b: Cysteamin supplementation during in vitro maturation and embryo culture: a useful tool for increasing the efficiency of bovine in vitro embryo production. Mol Reprod Dev 62: 203-209

Del Corso A, Capiello M, Mura U, 1994: Thiol dependent oxidation of enzymes: the last chance against oxidative stress. Int J Biochem 26: 745-750 
Deleuze S, Goudet G 2010: Cysteamine supplementation of in vitro maturation media: A review. Reprod Dom Anim 45: 476-482

Furnus CC, de Matos DG 1999: The availability of cysteine in culture media appears to be the limiting factor for gluthatione synthesis in mammalian oocytes. Theryogenology 51: 373

Guerin P, El Moutassim S, Menezo Y, 2001: Oxidative stress and protection against reactive oxygen species in preimplantation embryo and its surroundings. Hum Reprod Update 7: 175-189

Issels RD, Nagele A, Eckert KG, Wilmanns W 1988: Promotion of cysteine uptake and its utilization for glutathione biosynthesis induced by cysteamine and N-acetil-cysteine. Biochem Pharmacol 37: 881-888

Karadjole M, Getz I, Samardzija M, Matkovic M, Makek Z, Karadjole T, Bacic G, Macesic N, Vince S 2006: Influence of cysteamine supplementation during in vitro maturation of bovine oocytes on developmental rate and embryo quality. Slov Vet Res 43: 48-51

Lee CS, Koo DB, Fang N, Lee Y, Shin ST, Park CS, Lee KK 2000: Potent and stage-specific action of glutathione on the development of goat early embryos in vitro. Mol Reprod Dev 57: 48-54

Lim JM, Liou SS, Hansel W 1996: Intracytoplasmic glutathione concentration and the role of $\beta$-mercaptoethanol in preimplantation development of bovine embryos. Theriogenology 46: 429-439

Olson SE, Seidel GE 2000: Culture of in vitro produced bovine embryos with vitamin E improves development in vitro and after transfer to recipient. Biol Reprod 62: 248-252

Parchment RE 1991: Programmed cell death (apoptosis) in murine blastocysts: extracellular free radicals, polyamines and other cytotoxic agents. In Vivo 5: 493-500

Samardzija M, Karadjole M, Matkovic M, Cergolj M, Getz I, Dobranic T, Tomaskovic A, Petric J, Surina J, Grizelj J, Karadjole T 2006: A comparison of BoviPure ${ }^{\circledR}$ and Percoll ${ }^{\circledR}$ on bull sperm separation protocols for IVF. Anim Reprod Sci 91: 237-247

Takahashi M, Nagai T, Hamano S, Kuwayama M, Okamura N, Okano A 1993: Effect of thiol compound on in vitro development and intracellular GSH content of bovine embryos. Biol Reprod 49: 228-232

Van Langendonckt X, Morales H, Massip A, Dessy F 1998: Effect of hydrogen peroxide on in vitro development of bovine embryos. Theriogenology 49: 221

Wang X, Falcone T, Attaran M, Goldberg JM, Agarwal A, Sharma R 2002: Vitamin C and Vitamin E supplementation reduce oxidative stress-induced embryo toxicity and improve the blastocyst development rate. Fertil Steril 78: $1272-1277$

Yoshida M, Ishigaki K, Nagai T, Chikyu M, Prusel VG, 1993: Glutathione concentration during maturation and after fertilization in pig oocytes: relevance to the ability of oocytes to form male pronucleus. Biol Reprod 49: $89-94$ 